J. Phys. IV France 127 (2005) 193-197

(C) EDP Sciences, Les Ulis

DOI: $10.1051 /$ jp4:2005127030

\title{
Élaboration de couches minces de carbone par ablation laser femtoseconde pour application aux biomatériaux implantables
}

\author{
A.-S. Loir ${ }^{1}$, F. Garrelie ${ }^{1}$, C. Donnet ${ }^{1}$, J.-L. Subtil ${ }^{1}$, M. Belin ${ }^{2}$, B. Forest ${ }^{3}$, \\ F. Rogemond ${ }^{1}$ et P. Laporte ${ }^{1}$ \\ ${ }^{1}$ Laboratoire Traitement du Signal et Instrumentation, CNRS UMR 5516, Université Jean \\ Monnet, 10 rue Barrouin, Bâtiment F, 42000 Saint- Étienne, France \\ ${ }^{2}$ Laboratoire de Tribologie et Dynamique des Systèmes, CNRS UMR 5513, École Centrale \\ de Lyon, 36 avenue Guy de Collongue, BP. 163, 69134 Ecully Cedex, France \\ ${ }^{3}$ Centre Science des Matériaux et des Structures, CNRS UMR 5146, Département \\ Mécanique Physique et Interfaces, École Nationale Supérieure des Mines de Saint-Étienne, \\ 158 Cours Fauriel, 42023 Saint- Étienne Cedex, France
}

\begin{abstract}
Résumé. Des films de tetrahedral amorphous-Carbon (ta-C) ont été déposés, sous vide poussé, par ablation d'une cible de graphite avec un laser Ti : saphir (durée d'impulsion $170 \mathrm{fs}$, fréquence de répétition $1 \mathrm{kHz}$, énergie maximale par impulsion 1,5 mJ, longueur d'onde $800 \mathrm{~nm}$ ) sur substrats standard et sur biomatériaux (acier AISI 316L, polyéthylène à très haut poids moléculaire). Les propriétés de ces couches (structure, propriétés nanomécaniques et tribologiques) ont été caractérisées, en fonction des conditions d'élaboration, en examinant l'intérêt de l'utilisation d'un laser femtoseconde et leur capacité à satisfaire aux exigences spécifiques du domaine biomédical. Les propriétés d'adhérence des films ont été considérablement améliorées lors du dépôt sur des substrats en acier inoxydable préalablement préparés par décapage ionique in situ sous atmosphère d'argon. La surface hémisphérique d'une tête fémorale, en acier inoxydable, de prothèse de hanche de diamètre 22,2 $\mathrm{mm}$ a été revêtue d'un film de DLC adhérent et homogène en épaisseur. La résistance à l'usure de ce revêtement sera quantifiée à l'aide d'un simulateur de marche durant un million de cycles (correspondant à une année d'activité physique d'un être humain).
\end{abstract}

\section{INTRODUCTION}

Le dépôt par ablation laser (Pulsed Laser Deposition, PLD) est une technique d'élaboration de couches minces qui s'est considérablement développée depuis plus de 10 ans. Elle permet l'obtention de couches de très haute pureté déposées sur des substrats dont la température pendant le dépôt peut rester proche de l'ambiante, en comparaison des techniques concurrentes. Il est ainsi possible d'envisager des dépôts sur des substrats sensibles aux élévations de température, en particulier les polymères organiques. Son utilisation s'est largement répandue pour le dépôt d'une grande variété de couches minces, s'étendant des oxydes au dépôt de DLC (Diamond-Like Carbon) [1]. Dans le cas spécifique des DLC, il a l'avantage additionnel de permettre le dépôt de films non-hydrogénés avec une majorité d'atomes de carbone hybridés $s p^{3}$ [2]. Le dépôt par ablation laser est généralement conduit avec des lasers de durée d'impulsion nanoseconde. L'apparition récente des lasers de durée d'impulsion femtoseconde apparaît prometteuse pour produire des couches minces de haute qualité. Si de nombreux travaux ont été consacrés au dépôt de couches minces par ablation laser nanoseconde, peu de travaux sont en revanche consacrés au dépôt de couches minces de carbone par ablation laser femtoseconde [3-7]. Les premiers travaux sont apparus en 1999, et cette technique connaît actuellement un développement conséquent. Les films de ta-C (tetrahedral amorphous-Carbon) sont devenus un enjeu industriel et économique important. Leurs domaines d'application sont très larges en électronique et en optique mais également en mécanique pour des applications technologiques exigeantes, comme par exemple l'automobile (injecteurs pour moteurs à 
rampe commune). Ce travail a pour objet le domaine biomédical notamment les applications concernant les biomatériaux implantables (prothèses de hanche). L'intérêt des DLC dans ce domaine réside dans leur bonne résistance à l'usure et leur faible coefficient de frottement couplés à une biocompatibilité et une activité antibactérienne [8]. Cet article est basé sur de précédents travaux et nous présentons ici les principaux résultats des caractérisations structurales, nanomécaniques et tribologiques des films déposés par ablation laser femtoseconde en se focalisant plus particulièrement sur les exigences spécifiques du domaine biomédical (rugosité, adhérence, ... ). Les dépôts réalisés sur des substrats en acier montrent une adhérence insuffisante pour l'application visée. Un décapage ionique in situ sous atmosphère d'argon a été mis en place pour la préparation de surface de ces substrats. Un bref résumé des résultats d'adhérence des revêtements déposés sur ces substrats «nettoyés » sont ensuite exposés. Nous présentons enfin la réalisation d'un film de DLC sur une tête sphérique en acier AISI 316L de prothèse de hanche de diamètre $22,2 \mathrm{~mm}$.

\section{CARACTÉRISATIONS DES FILMS DE DLC}

Des films de DLC ont été déposés sur des substrats standard (silicium) et des biomatériaux (acier inoxydable austénitique AISI 316L et polyéthylène à très haut poids moléculaire) par ablation laser femtoseconde d'une cible de graphite de haute pureté $(99,997 \%)$ dans une enceinte ultra-vide à température ambiante [9]. Le laser utilisé est un laser saphir : Ti (Concerto, TCL) de longueur d'onde $800 \mathrm{~nm}$, délivrant une énergie par impulsion de $1.5 \mathrm{~mJ}$ (permettant ainsi d'obtenir des densités de puissance sur la cible de $10^{14} \mathrm{~W} / \mathrm{cm}^{2}$ ) avec une durée d'impulsion de $170 \mathrm{fs}$ à un taux de répétition de $1 \mathrm{kHz}$. Le détail du dispositif expérimental ainsi que la préparation des échantillons ont déjà été mentionnés par ailleurs [9].

Durant le processus de dépôt, différentes intensités laser peuvent être atteintes en modifiant la focalisation $\mathrm{du}$ faisceau sur la cible. Les autres paramètres sont maintenus constants de manière à étudier l'effet de la fluence laser sur les propriétés des couches obtenues notamment leurs propriétés tribologiques et nanomécaniques. Les valeurs des fluences laser utilisées pour les dépôts, ainsi que les principales propriétés des couches minces obtenues, sont reportées dans le tableau 1 .

Tableau 1. Caractéristiques et propriétés des couches minces de DLC déposées sur des substrats en silicium [9].

\begin{tabular}{cccccccc}
\hline \hline & $\begin{array}{c}\text { Fluence } \\
\text { laser } \\
\left(\mathrm{J} / \mathrm{cm}^{2}\right)\end{array}$ & $\begin{array}{c}\text { Epaisseur } \\
(\mathrm{nm})\end{array}$ & $\begin{array}{c}\text { Pourcentage } \\
\text { de carbone } \\
\mathrm{sp}^{3}(\%)\end{array}$ & $\begin{array}{c}\text { Dureté } \\
(\mathrm{GPa})\end{array}$ & $\begin{array}{c}\text { Module } \\
\text { d'Young } \\
(\mathrm{GPa})\end{array}$ & $\begin{array}{c}\text { Contraintes } \\
\text { intrinsèques } \\
(\mathrm{GPa})\end{array}$ & $\begin{array}{c}\text { Coefficient } \\
\text { de frottement } \\
\text { (air ambiant) }\end{array}$ \\
\hline $\mathrm{A}$ & 1,3 & $250-300$ & 71 & $20 \pm 2$ & $240 \pm 29$ & $-0,8$ & $0,1-0,2$ \\
$\mathrm{~B}$ & 2,8 & $120-150$ & 73 & $25 \pm 2$ & $260 \pm 10$ & $-1,8$ & $0,1-0,2$ \\
$\mathrm{C}$ & 5,2 & $85-95$ & 70 & $18 \pm 1$ & $210 \pm 13$ & $-2,8$ & $0,1-0,2$ \\
\hline \hline
\end{tabular}

La spectroscopie NEXAFS (Near Edge X-ray Absorption Fine Structure) réalisée sur les dépôts obtenus à différentes fluences laser permet de remonter au taux d'hybridation $s p^{3}$ des atomes de carbone par intégration des pics $\pi^{*} s p^{2}$ et $\sigma^{*} s p^{3}$ du graphite et du diamant respectivement [9-11]. La fluence laser, dans la gamme étudiée, ne semble pas affecter de manière significative la nature du DLC en terme d'hybridation du carbone. Les couches minces de DLC montrent un pourcentage moyen de l'ordre de $70 \%$ d'atomes de carbone hybridés $s p^{3}$ ce qui permet de les classer parmi les films de ta-C [12].

Les contraintes résiduelles sont, de manière très significative, faibles par rapport aux valeurs observées dans des couches DLC non dopées et sans sous-couches d'accrochage. Dans la gamme de fluences laser étudiée, les films déposés montrent des contraintes résiduelles compressives variant de -0,8 à -2,8 GPa, ce qui est inférieur aux niveaux de contraintes observées par exemple sur des couches ta-C obtenues par ablation laser en mode nanoseconde [13,14]. Ces faibles niveaux de contraintes résiduelles représentent un avantage majeur de l'ablation laser en mode femtoseconde, par rapport au mode nanoseconde. 
Perrière et al. ont également observé une tendance similaire, en comparant des couches de ZnO déposées par ablation laser femtoseconde et nanoseconde [15].

Les duretés et les modules d'élasticité en fonction de la profondeur d'indentation ont été mesurés pour les trois fluences laser déjà mentionnées. Les résultats sont reportés dans le tableau 1. Les valeurs atteignent un maximum pour une fluence laser de $2,8 \mathrm{~J} . \mathrm{cm}^{-2}$ avec une dureté de $25 \mathrm{GPa}$ et un module d'Young de $260 \mathrm{GPa}$. Ces résultats sont relativement modestes comparés à ceux obtenus par ablation laser en régime nanoseconde [2,16]. Cependant, ces valeurs sont tout à fait conformes à celles de la littérature [17] compte tenu du taux d'hybridation $s p^{3}$ mesuré dans nos couches.

Les couches obtenues se caractérisent par des coefficients de frottement faibles $(0,1-0,2)$ et stables sur de longues périodes (50 000 cycles). A la suite de ces 50000 cycles, des mesures profilométriques des dimensions des traces d'usure ont permis de déterminer des coefficients d'usure très faibles de l'ordre de $10^{-8}-10^{-9} \mathrm{~mm}^{3}$. $(\mathrm{Nm})^{-1}$. Ces valeurs classent ces couches de ta-C déposées par ablation laser femtoseconde parmi les matériaux à haute résistance à l'usure publiées dans la littérature [18].

\section{AMÉLIORATION DE L'ADHÉRENCE DES FILMS}

Quel que soit le procédé de dépôt utilisé, les films de DLC déposés sur des substrats en acier inoxydable présentent des propriétés d'adhérence très médiocres [19-21]. Les revêtements utilisés pour des applications biomédicales, notamment concernant des utilisations tribologiques et mécaniques doivent présenter une bonne adhésion à la surface du substrat. Dans le but d'améliorer l'adhérence des films de ta-C déposés sur les substrats en acier inoxydable, nous avons mis en place un dispositif de décapage ionique in situ sous atmosphère d'argon, décrit par ailleurs [22], afin de pulvériser les couches d'oxydes présentes en surface de ces substrats. L'épaisseur de la surface abrasée, pour une durée de décapage de 5 minutes, est de l'ordre de $0,01 \mu \mathrm{m}$. La rugosité de surface $\mathrm{R}_{\mathrm{a}}$ mesurée est de l'ordre de $20 \mathrm{~nm}$ et n'est pas modifiée par cette préparation de surface. La force d'adhésion des films déposés sur substrats non décapés et décapés a été mesurée à l'aide de tests de traction afin de montrer l'apport du décapage ionique préalable au dépôt [22]. Les revêtements élaborés sur substrats non décapés montrent de faibles valeurs d'adhérence. Concernant les films déposés sur substrats décapés, lors du test de traction, une rupture cohésive au sein de la colle est observée quelle que soit la fluence laser utilisée. La force d'arrachement mesurée, dans ce cas, permet d'estimer des valeurs de force d'adhésion supérieures à $45 \mathrm{MPa}$. Le décapage ionique in situ des substrats en acier inoxydable a multiplié les valeurs d'adhérence des films de DLC par un facteur 2.

Des tests tribologiques ont ensuite été effectués, dans les mêmes conditions que précédemment, sur des films de ta-C déposés sur des substrats en acier préalablement décapés. Les couches minces réalisées montrent un coefficient de frottement faible et stable de l'ordre de 0,1-0,2 et des coefficients d'usure de l'ordre de $10^{-9} \mathrm{~mm}^{3}$. $(\mathrm{Nm})^{-1}$. Le décapage ionique in situ effectué préalablement au dépôt ne semble pas affecter les propriétés tribologiques des films de ta-C déposés par ablation laser femtoseconde.

\section{REVÊTEMENT D’UNE PROTHÈSE DE HANCHE}

Le but de cette étude est de déposer des films de DLC sur une tête sphérique d'une prothèse de hanche de 22,2 mm. Nous avons donc réalisé des dépôts hors-axe (par rapport à l'axe de la cible) sur des substrats plans de « grandes dimensions » (de l'ordre de $20 \mathrm{~cm}^{2}$ ) afin d'obtenir des films de DLC homogènes en épaisseur [22]. A partir de ces conditions expérimentales, un système de rotation du substrat, incliné avec un angle de $45^{\circ}$ (figure 1), a été spécialement conçu.

Après un décapage ionique in situ préalable au dépôt dans les mêmes conditions que précédemment, une tête fémorale hémisphérique de diamètre $22,2 \mathrm{~mm}$ a été revêtue, par dépôt hors-axe, d'un film de carbone amorphe adhérent et homogène en épaisseur. Deux têtes sphériques de prothèses de hanche, non revêtue et revêtue d'un film de DLC, sont présentées sur la figure 2. 


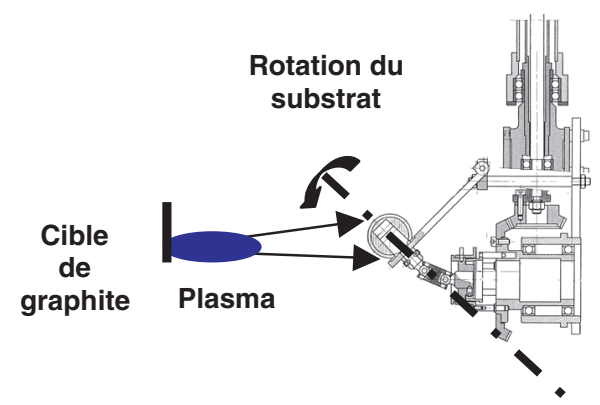

Figure 1. Principe du revêtement d'une tête fémorale de prothèse de hanche de diamètre $22,2 \mathrm{~mm}$.

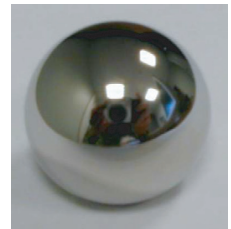

a) Tête sphérique vierge

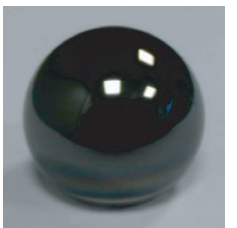

b) Tête sphérique revêtue d'un film de DLC après un décapage ionique in situ

Figure 2. Photographies optiques d'une tête sphérique de prothèse de hanche avant et après dépôt d'un film de DLC.

La résistance à l'usure de la tête sphérique revêtue contre une cupule en polyéthylène à très haut poids moléculaire sera ensuite étudiée dans une solution de Ringer (solution physiologique artificielle) sur un simulateur de marche durant plusieurs mois. Des essais tribologiques préliminaires (pion à mouvement alterné) réalisés dans une solution de Ringer durant 10000 cycles sous conditions sévères (pression de Hertz de 0,5 GPa) ont montré des coefficients de frottement encourageants de l'ordre de $0,04-0,05$.

\section{CONCLUSION}

Le travail présenté dans cet article a pour objectif le revêtement d'une tête sphérique de prothèse de hanche afin de prévenir les problèmes d'usure et de repousser dans le temps une éventuelle réintervention sur le patient. Des caractérisations nanostructurales, mécaniques et tribologiques des films élaborés sur des substrats standards de silicium ont été menées systématiquement pour trois valeurs de fluence laser de référence (environ 1,4, 2,8 et 5,2 J.cm ${ }^{-2}$ ) situées dans la gamme disponible de la source laser de durée d'impulsion femtoseconde. La forte proportion d'atomes de carbone hybridés $\mathrm{sp}^{3}$ permet de classer les films élaborés parmi les films de ta-C. L'ensemble des résultats permet de confirmer les bonnes dispositions des films de ta-C pour l'application prothèse de hanche, finalité de ce travail. L'adhérence des films de DLC a été déterminée sur des substrats d'intérêt biomédical. L'adhérence des revêtements sur des substrats en acier AISI 316L s'est révélée insuffisante. Un décapage ionique in situ sous atmosphère d'argon de ces substrats (préalable au dépôt) a alors été effectué avec pour objectif une amélioration de cette faible adhérence. Il a été montré que cette préparation de surface in situ multipliait par un facteur 2 l'adhérence des films sur les échantillons métalliques et n'affectait pas la résistance à l'usure du matériau déposé. Par ailleurs, des films de DLC présentant une répartition homogène en épaisseur ont été réalisés sur des substrats de « grandes dimensions » c'est-à-dire compatibles avec l'application visée. Des dépôts de DLC sur des pièces prototypes ont enfin été réalisés après un décapage ionique in situ des échantillons. Des tests complémentaires tels que le test d'usure sur simulateur de marche ou le test de résistance à 
la stérilisation restent à effectuer afin de confirmer les aptitudes des films de DLC élaborés par ablation laser femtoseconde pour l'application prothèse de hanche.

\section{Remerciements}

La présente étude est réalisée avec le soutien financier de la Région Rhône-Alpes (Thématiques Prioritaires), du Conseil Général de la Loire et avec le concours de l'Union Européenne (Fonds FEDER) dans le cadre du Programme de Reconversion Régionale et Sociale 1997-1999. Les auteurs remercient également P. Masschelein (LTSI, SaintEtienne) pour les analyses par profilométrie et C. Boachon pour les tests d'adhérence.

\section{Bibliographie}

[1] Chrisey D.B. et Hubler C.K., Pulsed Laser Deposition of Thin Film (Editions Wiley \& Sons, New York, 1994).

[2] Voevodin A.A. et Donley M.S., Surf. Coat. Technol. 82 (1996) 199-213.

[3] Qian F., Craciun V., Singh R.K., Dutta S.D. et Pronko P.P., J. Appl. Phys. 86 (1999) 2281-2290.

[4] Banks P.S., Dinh L., Stuart B.C., Feit M.D., Komashko A.M., Rubenchik A.M., Perry M.D. et McLean W., Appl. Phys. A 69 (1999) S347-S353.

[5] Okoshi M., Higuchi S. et Hanabusa M., J. Appl. Phys. 86 (1999) 1768-1770.

[6] Garrelie F., Jonin C., Baubeau E., Audouard E., Laporte P., Catherinot A. et Champeaux C., «Femtosecond Laser Ablation of Graphite: Study of the Plasma Plume and Thin Films Deposition », CLEO CFK5, 7-12 Mai 2000, pp. 615.

[7] Yao D.S., Liu J.-R., Wang L.-G., Yu C.-X. et Zhan R.-J., Chin. Phys. Lett. 17 (2000) 540-541.

[8] Grill A., Diamond Relat. Mater. 12 (2003) 166-170.

[9] Garrelie F., Loir A-S., Donnet C., Rogemond F., Le Harzic R., Belin M., Audouard E. et Laporte P., Surf. Coat. Technol. 163-164 (2003) 306-312.

[10] Pappas D.L., Saenger K.L., Bruley J., Krakow W. et Cuomo J.J, J. Appl. Phys. 71 n¹1 (1992) 5675-5684.

[11] Koivusaari K.J., Levoska J. et Leppävuori S., J. Appl. Phys. 85 n5 (1999) 2915-2920.

[12] Robertson J., Mater. Sci. and Eng. R: Reports, 37 (2002).

[13] Voevodin A.A., Donley M.S. et Zabinski J.S., Surf. Coat. Technol. 92 (1997) 42-49.

[14] Grill A., Diamond Relat. Mater. 12 (2003) 166-170.

[15] Perrière J., Millon E., Seiler W., Boulmer-Leborgne C., Craciun V., Albert O., Loulergue J.C. et Etchepare J., J. Appl. Phys. 91 n² (2002) 690-696.

[16] Bonelli M., Fioravanti A.P., Miotello A. et Ossi P.M., Europhys. Lett. 50 n 4 (2000) 501-506.

[17] Shi X., Flynn D., Tay B.K., Prawer S., Nugent K.W., Silva S.R.P., Lifshitz Y. et Milne W.I., Philos. Mag. B 76 (1997) 351-361.

[18] Erdemir A. et Donnet C., Modern Tribology Handbook (B. Bhushan CRC Press LLC, Boca Raton, 2000) 2 chpt 24 p. 871.

[19] Morshed M.M., McNamara B.P., Cameron D.C. et Hashmi M.S.J., Surf. Coat. Technol. 163-164 (2003) 541-545.

[20] Tsui Y.Y. et Redman D.G., Surf. Coat. Technol. 126 (2000) 96-101.

[21] Hou Q.R. et Gao J., Appl. Phys. A 68 (1999) 343-347.

[22] Loir A-S., Garrelie F., Donnet C., Belin M., Forest B., Rogemond F. et Laporte P., Thin Solid Films 453-454 (2004) 531-536. 